\title{
La crête de l'incendie Relecture des Lettres à Fouad El-Etr sur le Romantisme allemand
}

\author{
Alexis Nuselovici (Nouss)*
}

\author{
Toute couleur, toute vie \\ naît d'où le regard s'arrête \\ Ce monde n'est que la crête \\ d'un invisible incendie. \\ PH. JaccotTet
}

\begin{abstract}
Alors que je suis penché sur ces lignes parvient l'annonce de la disparition de Philippe Jaccottet. Étrange phénomène que celui-là, l'information, brutalement surgie dans la sphère publique, de la mort d'une personnalité sans que ne soit encore dissipé le lien entre l'histoire et le présent : un artiste, un penseur, un écrivain, un politicien - resteront-ils dans la mémoire ou s'effaceront-ils avec l'actualité ?Quelques mots portés par une voix anonyme ou une ligne imprimée font, de manière inversement proportionnelle à l'importance de l'énoncé, prendre conscience d'une place occupée par le disparu dans la mémoire individuelle ou la culture collective. Commence alors le travail de la survie, parallèle au travail de deuil pour les intimes et parfois complémentaire, la survie ou survivance (überleben ou fortleben), concept dédoublé dont on sait qu'il est central dans la pensée de Walter Benjamin sur la traduction et que commenta Antoine Berman dans le cahier 3 de L'Âge de la traduction où il traduisait les termes par survie et continuation.
\end{abstract}

De la survie/continuation de l'œuvre d'Antoine Berman, le présent numéro est à la fois témoignage et illustration. Mais une œuvre ne survit

* Aix Marseille Université, CIELAM, Aix-en-Provence 
jamais seule pas plus qu'elle ne surgit jamais seule. Parce qu'elle ne peut se réfugier dans la singularité et l'autonomie du vivant, une œuvre survivante en côtoie d'autres, les traces s'entrelacent, les images se mêlent. De même pour les humains. Berman et Jaccottet ce soir comme auparavant Berman et Meschonnic, Berman et Benjamin, Berman et Novalis puisque je reprends ici Lettres à Fouad El-Etr sur le Romantisme allemand. Berman et Jaccottet justement unis dans la fascination pour le romantisme allemand. Berman empruntant à Hölderlin le titre de L'épreuve de l'étranger et Jaccottet sous la direction duquel est publié le volume de La Pléiade consacré à l'auteur d'Hypérion et au traducteur d'Antigone. Les deux tracés soudainement se confondent, sous le signe double de la poésie et de la traduction.

Il est un romantisme allemand français, comme il est un Kafka français, comme il est un Bartleby français, comme il est un « Donne français » selon Antoine Berman - non pas une appropriation ou une manipulation (quoique cela puisse correspondre à un travers des Français, fort de leur égouniversalisme congénital) mais une traduction, légitime en cela, la puissance d'un original se mesurant de la gamme possible de ses lectures. Plus précisément, une traduction/translation, dans les termes d'Antoine Berman, afin d'exprimer l'ampleur du passage, du transfert. Entre littérature et philosophie - ce qui déjà le caractérise -, plusieurs mouvances, générationnelles ou non composent ce R.A.F.1 : le romantisme d'Albert Béguin, de Marcel Brion, de Maurice Blanchot, d'Armel Guerne, de Jean-Luc Nancy et Philippe Lacoue-Labarthe. Celui d'Antoine Berman est particulier en ce que d'emblée il pose la question : «Comment écrire romantiquement sur le Romantisme, mystérieusement sur le mystère, fragmentairement sur le fragment? J'ai eu peur, soudain, de me trouver enfermé dans la prison de l'entendement et de la critique » (p. 9) et en ce qu'il écrit romantiquement en prolongeant la question tout au long du livre sous la suite d'intuitions intellectuelles - pour reprendre l'expression de F. Schlegel qui en fait l'impératif catégorique de la théorie - et dans le fil des propositions critiques. Ses écrits ultérieurs ne s'enfermeront jamais dans le dogme ou la

\footnotetext{
${ }^{1}$ R.A.F. : romantisme allemand français. Qu'on me permette ce plaisir ludique - pas éloigné d'un humour romantique -, proche du plaisir nominatif consistant, par réconfortante familiarité, à désigner dans ce texte les titres en abrégé : Lettres, L'épreuve, L'auberge, le John Donne, le Jacques Aymot.
} 
prescription : là où d'autres proposent des stratégies ou des principes de traduction, il dégage des «forces" ou des «tendances déformantes" (L'auberge). Cet aveu initial explique que la démarche assertive, chez Antoine Berman, n'est jamais définitive ou close, que par exemple, L'épreuve se conclut par une section - annoncée par le prologue, «La traduction au manifeste »-découpant ce que serait « la traduction comme nouvel objet de savoir ", un domaine à fonder ou à creuser sur des données existantes. L'appel a été entendu, partiellement.

La visée qu'il reconnaissait dans le romantisme allemand - la quête d'un absolu et la conviction de l'inachèvement nécessaire, double bind conceptuel et formel - accompagne son propre cheminement et éclaire la nature de l'œuvre, éclatée, hétéroclite car L'épreuve, L'auberge, Traduction et recherche scientifique (que je n'ai pas lu), le John Donne forment un ensemble pour le moins disparate, un territoire d'écriture qu'une cartographie ne saurait ordonner, sans compter les articles, séminaires et communications. Il faudrait au demeurant considérer similairement son œuvre de traduction, en globalité sans trop faire la part des travaux dits alimentaires car la déontologie (le sérieux) rejoint ici l'éthique (le respect) et, après tout, une traduction est une traduction: Novalis, Schlegel, Roa Bastos, Artl, Schleiermacher, Scorza, P. Härtling, G. Manzur, entre autres, sans compter les auteurs non-littéraires en sociologie, histoire ou géographie. La survie est le mode de la vie sous le régime de l'inachevé. Impossible ici de ne pas remarquer que la production d'Antoine Berman est largement posthume (John Donne, L'auberge, Jacques Amyot...), ce qui oblige le lecteur, comme pour Kafka ou Pessoa, à moduler sa lecture en tenant compte de ce qui l'autorise. Insérer l'absence dans le présent d'une lecture. Les romantiques non plus n'avaient peur ni de la mort ni de la nuit.

Centralité de «l'imparfait» qui empêche de considérer Lettres comme fondateur au sens où il contiendrait toute l'œuvre à venir comme souvent une critique avide de fondations aime à traiter les ouvrages initiaux. L'exercice serait facile puisque le romantisme allemand dessine l'axe de référence majeur pour Antoine Berman, comme le montrera L'épreuve mais ce serait erroné puisque «le romantisme est un mouvement au sens le plus strict » qui « ne peut s'affirmer que par une perpétuelle multiplication » (p. 
19). L'inachevé suggère un mode d'écriture et un mode de pensée à la fois parce qu'il garantit l'universel en tant que totalité non fermée et le progrès en tant que quête permanente et parce qu'il proclame une injonction quant au travail spéculatif : «L'horreur de l'impensé, voici la faute de toute pensée. Il y a une volonté du savoir de tout représenter, de tout porter à la clarté, à sa clarté, pour laquelle l'impensé apparaît nécessairement comme quelque chose de négatif. Mais l'impensé témoigne des limites essentielles de la pensée, des limites qu'elle doit respecter pour rester une pensée de la pensée des choses » (p. 42). L'impensé, autre nom de l'intraduisible.

Ce qui est commun à tous les exposants du R.A.F. est l'insistance sur la fulgurance créatrice et l'intensité biographique des auteurs qui les a souvent placés dans une position de marginalité ou de minorité qui garantissait leur liberté. Ne jugera-t-on pas qu'Antoine Berman a pu jouir d'un privilège semblable en demeurant à l'écart du système universitaire (à l'instar de Walter Benjamin), même s'il en a souffert et que nombreux sommes-nous à y voir un scandale? Lorsqu'à partir de Novalis, il loue « cette fluidité primordiale $[. .$.$] qui désigne la région profonde du Romantisme » et$ affirme « la liberté intime de tout ce qui est liquide : eau, lait, mercure... » (p. 27), ne rejoint-il pas l'argument deleuzien en faveur des lignes de fuite qui garantissent la déterritorialisation, le devenir, la création ? Deleuzien, Antoine Berman le serait encore lorsqu'il aborde le romantisme allemand comme un écrire-vivre-penser, un complexe affirmant sa modernité par ce continuum rappelant la séquence affect/percept/concept développé dans Qu'est-ce que la philosophie ? Si Foucault est directement mentionnée dans l'œuvre par l' «archéologie » nécessaire de la traduction, Deleuze semble absent mais il appert que le rapport d'Antoine Berman à l'espace philosophique qui lui était contemporain passe par un dialogue muet, que cet espace se glisse dans «l'espace de l'âme» (p. 20), «l'étendue totale de l'âme» (p. 34), ouverte à tout ce qui l'entoure, défiant les catégories et les contradictions en vertu d'un « principe de passage universel» (p.44) ou de «versabilité infinie » (p. 32), l' « encyclopédie », à la fois le rêve et le projet des romantiques, non pas un savoir rigide et monumental mais un système à produire « des "pensées" fluides, mouvantes et presqu'insaisissables (sic) dans leur éloignement » (p. 42). 
Dans sa disparité, l'œuvre d'Antoine Berman ne suggère dès lors pas un éparpillement mais une dissémination: "Tout est germe » dit un fragment de Novalis, ce que suggère l'image de la harpe éolienne, du même, reprise par Walter Benjamin dans son essai sur la traduction. Les sons se répondent les uns autres comme le font les pensées, comment le font les phrases, comme le font les êtres, composant une immense trame de résonances et d'analogies, la «sympoésie ». Loin de l'image blafarde d'un romantisme désespéré, celui-ci se retrouve positivé, une entreprise de connaissance non totalisante, une saisie du monde menée selon une exigence éthique et définissant cette exigence, ce qu'évidemment développera L'épreuve.

Le romantisme s'affirme dans son cheminement entre le présent et le permanent, entre le concret et l'idéal, et non immobilisé sur le second versant comme certains esprits frileux le veulent. Son entreprise consiste à trouver un sens de l'histoire qui ne se réduise pas à la réduction rationaliste connue sous ce nom, sans profondeur et sans horizon. D'où sa préférence pour le terme $\mathrm{d}^{\prime}$ " âge » parmi ceux qui mesurent le temps et sa revendication de $l^{\prime}$ « Âge d'or » (p. 22, 30, 45). Il tranche sur « époque » ou « période » par une dimension organique, voire biologique, qui le déplace du côté du vivant, qui lui donne la charge d'une promesse, reprise dans le titre, L'Âge de la traduction, qu'Antoine Berman donnera à son commentaire de Walter Benjamin. (Opportune actualité : le covid a ouvert, de l'opinion d'experts, l'âge des pandémies - ne sont-elles pas des traductions virales universelles?)

Parmi tous les thèmes mis en relief, celui du fragment est prédominant, comme le plus apte à paradigmatiquement figurer le romantisme allemand. Loin de revêtir une valeur exemplaire, il déploie une force matricielle dont Antoine Berman liste les formes: «esquisses, brouillons, essais, études, remarques, dialogues, notes, discours, anecdotes, proses et poésies mêlés » (p. 43). Ne manquerait-il pas deux formes que leur absence rend terriblement évidentes ? La lettre et la traduction. En dépit de son cadrage rhétorique - et qui fait son charme identificatoire (le lieu, la date, l'heure, «Mon cher », «Bonne nuit ») -, la forme de la lettre appartient au fragment et à l'essai. Schlegel faisait du dialogue un enchainement de fragments et de la correspondance un dialogue étendu. Délibérément 
inscrite dans le temps, la lettre pourtant le défait puisqu'elle s'insère dans une chronologie parallèle ou parasite. Elle suscite comme un temps autre, l'équivalent d'un temps sacré que l'on retrouve en abandonnant le temps des horloges. Le lecteur y accède lorsqu'il lit Lettres dans l'indifférence de ce que ces textes soient passés par une enveloppe et un timbrage postal ; il lit un livre arborant ce titre. La lettre-à (un jeune poète ou à Fouad El-Etr) forme un genre littéraire qu'il ne faut pas confondre avec la correspondance (Madame de Sévigné ou Flaubert) ou le roman épistolaire (Liaisons dangereuses ou Lettres persanes) ; son histoire va de l'épitre (antique ou du XVIIe siècle) à Derrida ou au blog. Or, elle entretient un rapport très étroit avec la traduction en ce que celle-ci suppose aussi une séquentialité (le texte original en amont et une inévitable retraduction possible en aval) qui creuse aussi un écart par rapport au temps calendaire. Ainsi, la lettre et la traduction relèvent d'une rhétorique du fragment, ce qu'Antoine Berman nommera « écrituretorse ou germe » dans $L^{\prime} \hat{A} g e$ (p. 93) et qu'il abordera au travers de « la copia ou principe d'abondance » dans le Jacques Amyot.

Une tonalité curieuse parcourt le livre, donnée par la référence récurrente à une circonstance militaire qui oblige l'auteur à garder la maison et à attendre la venue d'un courrier ${ }^{2}$ qui arrivera dans les dernières lignes, ordonnant le baisser de rideau sur la scène épistolaire. Le réel colore ainsi l'écriture, celui-ci, administratif, mais non moins la pluie automnale ou le jaune mélancolique du soleil, fleur vivace cousine du tournesol. Comme dans toute correspondance, il y a de l'autoportrait dans Lettres, ce qui apparaît formellement par l'insertion dans l'écriture d'Antoine Berman de citations des romantiques, parfois non attribuées et jamais référencées, comme si la parole romantique se mêlait à la sienne, en osmose - un concept romantique. Ou comme si de leur parole à la sienne s'établissait un processus d'intertraduction. L'autoportrait, au demeurant, est une manifestation de la réflexivité qu'Antoine Berman cerne comme un autre concept éminemment romantique et dont il fera un aspect majeur de sa pensée de la traduction. Mais Lettres esquisse aussi un autre autoportrait, étalé, anamorphique qui

\footnotetext{
${ }^{2}$ Je dois à Isabelle Berman l'éclairage adéquat : Antoine Berman attendait sa convocation pour « les trois jours » auxquels l'armée française soumettait les futurs appelés au service militaire afin de leur attribuer une affectation ou de les réformer.
} 
dessinerait au travers des ouvrages le visage d'Antoine Berman avec, entre autres, ceux de Novalis et Schlegel, Amyot, Paz, Jaccottet, Bonnefoy.

Après tout, la pratique de l'autoportrait polymorphe est propre à tout traducteur lorsqu'il traduit, se redessinant à chaque fois dans le texte traduit à partir du texte original. Un narcissisme altruiste, en somme. Une bienveillance. Un respect. Le soi par l'autre. Reconnaître dans le romantisme « cette action qui veut instaurer - avec ou sans littérature - un ordre poétique communautaire, une sympoésie » (p. 23). Nous y sommes conviés, « entre le matin ivre et la légèreté du soir »(Ph. Jaccottet).

\section{Euvres citées}

BERMAN, Antoine. L'Épreuve de l'étranger. Culture et traduction dans l'Allemagne romantique. Paris : Gallimard, 1984 [L'épreuve].

BERMAN, Antoine. Lettres à Fouad El-Etr sur le Romantisme allemand. La Délirante, 1991 [Lettres/1968].

BERMAN, Antoine. Pour une critique des traductions : John Donne. Paris : Gallimard, 1995 [John Donne].

BERMAN, Antoine. La traduction et la lettre ou l'auberge du lointain. Paris : Seuil, 1999.

BERMAN, Antoine. L'Âge de la traduction. "La tâche du traducteur » de Walter Benjamin, un commentaire. Paris: Presses universitaires de Vincennes, 2008.

BERMAN, Antoine. Jacques Amyot, traducteur français. Essai sur les origines de la traduction en France. Paris : Belin, 2012.

JACCOTTET, Philippe. Poésie 1946-1967. Paris : Poésie/Gallimard, 1971.

JACCOTTET, Philippe. L'encre serait de l'ombre. Paris : Poésie/Gallimard, 2011. 


\section{Résumé}

Relire Lettres à Fouad El-Etr sur le Romantisme allemand, un des premiers écrits théoriques d'Antoine Berman, afin de tenter de comprendre sa dette, au sens de Walter Benjamin, à l'endroit de ce mouvement littéraire à la fois sur le plan de la pensée que des formes adoptées pour l'exprimer. Y reconnaître aussi les germes de concepts qui a accompagneront toute l'œuvre bermanienne, de l'éthique à la critique.

Mots-clés : Romantisme ; Survie ; Inachevé ; Poésie ; Traduction ; Critique ; Philosophie

\section{Resumo}

Reler Lettres à Fouad El-Etr sur le Romantisme allemand, um dos primeiros escritos teóricos de Antoine Berman, para tentar compreender sua dívida, no sentido de Walter Benjamin, com a posição desse movimento literário, tanto a respeito do pensamento quanto das formas adotadas para expressá-lo. Também, para tentar reconhecer ali os conceitos germinais que acompanharam toda a obra bermaniana, da ética à crítica.

Palavras-chaves: Romantismo; Sobrevida; Inacabado; Poesia; Tradução; Crítica; Filosofia 